\title{
RHEUMATOLOGY
}

\section{Pathogenesis of juvenile chronic arthritis: genetic and environmental factors}

\author{
Kevin Murray, Susan D Thompson, David N Glass
}

This is the eighth article in a series on rheumatology.

\author{
Division of \\ Rheumatology, \\ Children's Hospital \\ Medical Center, 3333 \\ Burnet Avenue, Pav \\ 2-129, Cincinnati, OH \\ 45229-3039, USA \\ K Murray \\ S D Thompson \\ D N Glass \\ Correspondence to: \\ Dr Glass.
}

Juvenile chronic arthritis (JCA) is a heterogeneous group of chronic arthropathies affecting approximately 1-3 children per 1000 . The initial oversimplified classification into pauciarticular, polyarticular, and systemic onset types has been expanded in a World Health Organisation-International League Against Rheumatism report to include seven different types of disease. ${ }^{1}$ It is clear that the majority of children with JCA have a different disease from adults with rheumatoid arthritis, although the chronic arthropathies have some common clinical and pathological features. As a group, these diseases are generally considered to be autoimmune in origin with the evidence supporting this varying between types. There is a general female preponderance, characteristic chronic inflammatory infiltrates in the synovium and immune responses characterised by autoantibodies including antinuclear antibodies (ANA), IgM rheumatoid factor, and antibodies to heat shock proteins and the oncoprotein DEK. In addition and in common with many other autoimmune diseases, there are extensive and well documented associations with genetic markers, primarily, but not exclusively, within the major histocompatibility complex (MHC) or HLA region on the short arm of chromosome 6 . It is anticipated that environmental triggering agents are also involved in the pathogenic process. In a recent review of a related disease, rheumatoid arthritis, the contribution of HLA genetics to pathogenesis was estimated to be approximately $37 \%$ with as yet unknown genes contributing further, with the balance being deemed largely due to environmental factors. ${ }^{2}$ The purpose of this review is to consider the relative contributions of genetic versus environmental factors as being causal in the various types of JCA.

\section{Genetic factors}

JCA is rarely familial so that a low index of suspicion for a simple genetic basis is in order. In early studies reported, Dr Barbara Ansell and Professor Eric Bywaters were able to identify family disease and concordance in twins. Subsequently multiplex families and sibling pair patients were reported. ${ }^{3}$ In the latter study, the 12 sibling pairs were biased towards pauciarticular onset type of disease and tended to be concordant for this type of JCA. It is noteworthy that those 12 pairs were drawn from a pool of over 2000 JCA patients under care. In a recent series of 60 sibling pairs, evidence strongly supportive of a genetic basis was obtained with a substantial concordance rate in sibling pairs (70-80\%) for the various types of JCA. ${ }^{5}$ The most common type of disease onset, pauciarticular or limited joint disease, was present in approximately 30 of 60 sibling pairs and the majority were concordant for both pauciarticular onset and course. Siblings had an average difference in age of onset of JCA of 4.5 years. Of greater interest, five affected twins concordant for pauciarticular JCA had onset of disease averaging only four months apart. The absence of concordance for type of JCA in sibling pairs would have mitigated very strongly against genetic factors in the pathogenesis of JCA; conversely, the presence of concordance is very consistent with a hereditary basis, but a role for additional environmental influences is by no means been excluded.

WHICH GENES ARE INVOLVED?

Given that these autoimmune arthropathies have at least a partial genetic basis, the candidate genes likely to be contributing to the process are those which are part of, or can influence the nature of, HLA binding of antigen and its presentation to $\mathrm{T}$ cell receptors (TCRs), central to the immune response, and thought to be integral to the pathogenesis of such arthropathies. ${ }^{6}$ The trimolecular complex so formed consists of an antigenic peptide presented on an HLA molecule which is recognised by a portion of the TCR called the complementarity determining region-3 (CDR3). The highly variable CDR3 sequence results from the TCR rearrangement process and is responsible for the specificity of antigen/HLA recognition. Such recognition leads to $\mathrm{T}$ cell clonal expansion, evidence of which is present in the JCA synovium. As a result of this process there are corresponding effects in both the macrophage (or antigen presenting cells bearing the HLA/antigenic peptide complex) and clonally expanded $\mathrm{T}$ cell populations, with the liberation of cytokines and a characteristic inflammatory cascade. Although the putative antigen(s) involved in the different forms of JCA have yet to be defined, substantial information is available about the other two members of the trimolecular complex. The present state of knowledge of HLA and TCR markers in JCA is summarised in table 1. 
Table 1 HLA and TCR associations with different forms of $\mathcal{F C A}$

\begin{tabular}{llll}
\hline Disease & Severity range & HLA association & TCR \\
\hline Pauciarticular JCA & + & $\begin{array}{l}\text { Class I + class II (A2) } \\
\text { (DR5, 6, 8, DP2) }\end{array}$ & V $\beta 20$ \\
Juvenile SpA & & Class I (B27) & V $\beta 20$ (?) \\
Systemic JCA & ++ & Class II (DR4) & - \\
Polyarticular JCA (RF-) & $+\rightarrow++++$ & Class II (DR8/DR3) & V $\beta 8,16$ \\
Polyarticular JCA (RF+)+(JRA) & +++ & Class II (DR1/DR4) & V $\beta 14,17$ \\
Rheumatoid arthritis (adult onset) & $++\rightarrow++++$ & Class II (DR1/DR4) & V $\beta 3,14,17$
\end{tabular}

$\mathrm{RF}=$ rheumatoid factor.

^ Juvenile onset spondyloarthropathy.

† Childhood onset of adult rheumatoid arthritis, or polyarticular juvenile rheumatoid arthritis (IgM RF+) in North American usage.

$\ddagger$ Clonal expansions identified in specific TCRV $\beta$ families.

MHC-HLA ASSOCIATIONS

These associations, summarised below, are well documented and described in detail elsewhere. ${ }^{78}$ The earliest reported association was with an MHC class I gene, HLA-B27 in older children with pauciarticular disease. ${ }^{9}$ There have since followed associations with most types of JCA, although in varying degrees of strength, some of which reflect similarities to adult arthropathies and others are unique to childhood. The HLA DR4 (DRB ${ }^{\star} 0401 /$ 0404/0405) association found in the $\operatorname{IgM}$ rheumatoid factor positive polyarticular JCA is equivalent to adult rheumatoid arthritis and is consistent with a role for the HLA DR1 $\left(\mathrm{DRB} 1^{\star} 0101\right)$ and DR4 shared epitope present in the third hypervariable region of the HLA DRB1 gene on these haplotypes. In contrast, the unusual features of the early pauciarticular onset JCA group, that is, the class I association with HLA A2 and various class II associations; -HLA DR5 (DRB1*11), DR8 $\left(\mathrm{DRB} 1^{\star} 0801\right)$, DR6 (DRB1*1301), and HLA $\mathrm{DPB}^{\star} 0201$, are unique to this arthropathy which affects primarily girls under the age of 6 . In addition this form of JCA is associated with a high frequency of a chronic iridocyclitis, ANA and anti-DEK autoantibodies. ${ }^{7}{ }^{10}$ The HLA associations reported in early onset pauciarticular JCA suggest at least three separate genetic effects within the MHC and that the three associations are interactive, that is, independent of each other and not dependent on linkage disequilibrium between alleles at the closely linked HLA loci. The complexity of the associations can be gauged from the increasing risk seen when considering all the known genetic variables. In pauciarticular JCA the risk for disease, compared with individuals without specific genes, can rise 10 -fold as multiple genetic variables, including gender, are considered. ${ }^{7}$ To date, no specific mechanisms have been identified as to how these genes are involved in disease induction. In addition there is some debate as to the relative importance of the closely linked HLA DR and HLA DQ loci. ${ }^{11}$ In support of this, one study has demonstrated a polymorphism in the $\mathrm{Y}$ box of the DQ promoter region associated with pauciarticular onset JCA. ${ }^{12}$ Another group has demonstrated limited linkage disequilibrium between the early onset pauciarticular JCA associated locus HLA-DPB ${ }^{\star}{ }^{\star} 0201$ and certain DQ/DR alleles, further highlighting the difficulties of identifying the true disease related gene. ${ }^{13}$
Given the normal function of HLA molecules in presenting processed antigenic peptides (both foreign and self) to effector $\mathrm{T}$ lymphocytes, as part of an immune or inflammatory reaction, it has been speculated that particular HLA risk alleles present potentially arthritogenic peptides to such T cells. An alternative hypothesis is that the HLA molecules themselves undergo antigen processing and become immunogenic when presented by other HLA molecule. This may explain in part how multiple independent HLA disease related alleles commonly exist in the one patient. Of great interest will be the nature of the peptides presented by the HLA molecules on antigen presenting cells in JCA patients. This may provide clues as to the pathogens involved especially if extrinsic antigens can be identified which have mimicry with self antigens. ${ }^{14}$ Although their specific role in immunopathogenesis has yet to be elucidated, it is clear that the 'at risk' HLA haplotypes influence disease outcome. For example, an HLA DR1 haplotype predicts a poor outcome with respect to arthritis in the pauciarticular onset group, but protects from eye disease, whereas the presence of HLA DR5 predicts more eye disease. ${ }^{15}$ In a similar manner a genetic polymorphism of the proteasomal subunit LMP2 (a non-HLA gene encoded in the MHC region on chromosome 6) has been associated with late onset pauciarticular JCA (B27 associated), especially those with more severe disease. ${ }^{16}$ At a nearby locus, also within the MHC region, a polymorphism in the TAP2 gene which encodes for a transporter associated with antigen processing (TAP2B), has been associated with early onset pauciarticular JCA. ${ }^{17}$ Both of these findings may implicate antigen processing either at a qualitative or quantitative level in disease susceptibility.

\section{T CELL RECEPTORS}

TCRs result from the rearrangement of gene segments in a similar fashion to immunoglobulin genes. This largely intrathymic process results in a repertoire of $\mathrm{T}$ cells expressing diverse TCRs which undergo selection to delete potentially self reactive $\mathrm{T}$ cells. Clonal expansion of specific $\mathrm{T}$ cells occurs locally in response to antigen and has been identified in the inflamed joints in JCA. Studies have shown that such clones can be present in multiple joints in the same individual, they persist over time and are specific to the joint, and rarely being seen in the peripheral blood lymphocyte population. ${ }^{18}$ Given the degenerate nature of the genetic code, several combinations of mRNA nucleotides can produce TCRs of identical amino acid structure. When looking at an extensive database of $\beta$ chain TCR sequences (CDR3 region) from children's joints, identical TCR amino acid sequences with different nucleotide sequences have been identified (S Thompson, unpublished observations). This CDR3 region is the site of specific interaction of the TCR with unique peptideHLA complexes. This recruitment and expansion of structurally identical clones is strongly indicative of an ongoing antigen driven immune 
response in the joint. That $\mathrm{T}$ cell clonal expansion is characteristic of the disease process is likely, but the extent to which genetic status influences the available TCR repertoire is less clear. In rheumatoid arthritis it has been suggested that the disease associated HLA DR1/DR4 haplotypes influence the available T cell repertoire during the thymic selection process perhaps permitting survival of potentially autoreactive $\mathrm{T}$ cells which are clonally expanded later in life in response to specific stimulation in the rheumatoid arthritis joint. ${ }^{19}$ Such a mechanism with HLA haplotypes different from rheumatoid arthritis could be involved in JCA. An additional mechanism for which there is some evidence, is the existence of individual null or deficient TCR genes, which are incapable of producing a functional TCR, and may predispose to autoimmune disease. Their association with diseases like $\mathrm{JCA}^{20}$ suggests a potential hole in the TCR repertoire preventing a specific required immune response. The association may alternatively be secondary to linkage disequilibrium with other normally functioning TCR genes involved in the pathogenesis of JCA.

NON-MHC, NON-TCR GENES

The spectrum of genes, many from outside the MHC complex and TCR regions which are putatively linked to or associated with autoimmune and inflammatory diseases is expanding rapidly. As a result, considerable genetic complexity is being added to the paradigm. Among the earliest genes associated with predisposition to rheumatic disease were complement deficiencies. Products of genes, which are primarily expressed in inflammatory cells such as macrophages or lymphocytes, both cell types prominent in the JCA synovium, have been implicated recently. Such markers include the natural resistance associated macrophage protein gene (NRAMP1) where a polymorphism has been linked to rheumatoid arthritis. ${ }^{21}$ Cytokine gene polymorphisms have also been identified with an interleukin (IL)-4 polymorphism linked to asthma ${ }^{22}$ and a tumour necrosis factor- $\alpha$ (TNF- $\alpha)$ polymorphism linked to autoimmune disease in general. The latter forms part of a known autoimmune associated HLA A1, B8, DR3 haplotype. Allelic differences in cytokine genes may influence the outcome of an immune response in a disease promoting manner as with $\mathrm{TNF}-\alpha$ where a polymorphism in the promoter region favours overproduction of TNF- $\alpha .^{23}$ In contrast an IL-4 promoter region polymorphism favours greater production of this cytokine which, while disease promoting in asthma, could conceivably be protective against chronic arthritis. In one study an IL- $1 \alpha$ allelic polymorphism was associated with pauciarticular onset JCA and chronic iridocyclitis. ${ }^{24}$ Such genetic polymorphisms which contribute to non-antigen specific or innate immunity may either influence susceptibility to specific infections or skew the immune response towards one cytokine profile or another (that is, Th1 $v$ Th2) and thus influence the outcome of an immune inflammatory response. ${ }^{25}$ Aberrant function of some of these genes may set the stage for unusual self or autoreactivity. Further influence upon the process may be related to the fate of autoreactive cells, which in normal individuals may be expected to be suppressed or undergo cell death through apoptosis. In this context, Fas and Fas ligand deficiencies have been implicated in experimental models as well as in human autoimmunity. ${ }^{26}{ }^{27}$ Animals transgenic for cytokine genes or with genes defective through knockout techniques can help establish novel candidate genes. For example, mice transgenic for the human TNF- $\alpha$ gene overproduce TNF- $\alpha$ and develop a polyarthritis. In contrast an autoimmune myopathy develops in TGF- $\beta$ (a potential anti-inflammatory cytokine) knockout mice. ${ }^{28}$ In these mice disease develops under germ free conditions, that is, in the absence of extrinsic antigen, suggesting self antigen presentation alone may be sufficient for disease. Another aspect of JCA, which is a feature of many autoimmune diseases, is the effect of gender. There is a particularly marked predisposition in pauciarticular JCA where females outnumber males seven or eight to one. The reason for such a sex bias is unknown but may relate to sex linked genetic factors involved in immune gene regulation.

JCA AS A COMPLEX GENETIC TRAIT

The sum of the evidence in JCA suggests independent roles for multiple different genes. Similar evidence for polygenic effects is accumulating in many autoimmune diseases including rheumatoid arthritis, insulin dependent diabetes mellitus and more common diseases such as psoriasis, hypertension, and asthma. The occurrence of disease and its phenotype is likely to be determined by complex genetic traits. Implicit in this concept is the likelihood that the genes involved are weakly penetrant, though in most instances necessary, for expression of the disease phenotype. Family history of the disease usually proves much weaker and atypical as compared with a fully penetrant monogenic disease with a typical mendelian inheritance pattern. Within the group of autoimmune diseases, insulin dependent diabetes mellitus, multiple sclerosis and rheumatoid arthritis, all have HLA loci associated with disease. JCA fits a similar model but the different types of JCA almost certainly have different genes implicated. Pauciarticular JCA, with at least two and perhaps three HLA loci involved, may already be considered a complex trait with respect to the HLA region alone. The complex trait hypothesis is important because it gives a framework for a comprehensive understanding of the pathogenic mechanisms involved as published studies in insulin dependent diabetes indicate. $^{29}$ Methodologies are now available (that is, gene markers) to study the entire genome and thus potentially locate all the specific chromosomal regions involved in each type of JCA. Although this approach, commonly based on the study of affected sibling pairs, is labour intensive, it is straightforward. Some of the difficulties of using families to study complex traits will be obviated, especially 
the problem of anticipating the family member who will develop disease in the future.

AUTOANTIGENS IN JCA

The self antigens thought to be driving autoantibody and $\mathrm{T}$ cell clonal expansion, characteristic features of autoimmune diseases including JCA, are currently being defined. Although the subject of considerable research, they are perhaps less well understood than the structural elements (HLA/TCR) involved in the antigen recognition events discussed above. Autoantibodies to nuclear components (ANA), IgM (rheumatoid factors), and collagen are not specific for JCA and occur in other autoimmune arthropathies. More recently, immune responsiveness to human heat shock proteins (HSP) has been associated with a relatively good outcome in joint disease in children. ${ }^{30}{ }^{31}$ Again, such immune reactivity is not limited to JCA and its role in the inflammatory process needs to be defined. In clinical terms, ANA and rheumatoid factor do have some validity as clinical markers for pauciarticular and late onset poly types of JCA respectively. ANA have also been associated with the iridocyclitis of JCA, although changing methodologies in the detection of ANA may, to some degree, alter the specificity of associations. In contrast to the above, recently identified antibodies to the nuclear protein DEK (a product of the putative oncogene $d e k$ ), show some evidence of specificity for pauciarticular JCA and its associated iridocyclitis. ${ }^{10}$

\section{Environmental component}

Classical epidemiological studies have not generally shown clustering of JCA patients that would imply a definite infectious aetiology, although some seasonality in systemic onset disease has been reported. Recently documented long term trends for the prevalence (or incidence) of JCA to change over time may also signify an environmental rather than a genetic effect. ${ }^{32}$ Several potential pathogens have been implicated but have not yet been shown to be definitely causal. Rubella virus has been implicated for some time and a small proportion of individuals who received rubella immunisation develops arthritis very similar to pauciarticular JCA. Sequence similarities have been shown between HLA DRB1*0801, DRB1*11 and DPB $1^{\star} 0201$, all associated with pauciarticular JCA, and an Epstein-Barr virus protein. ${ }^{14}$ This may represent an example of how molecular mimicry between self and non-self proteins has a role in JCA, and may also define a role for the involvement of specific HLA genes associated with the disease.

Chlamydia has been identified in the joints of some children with arthritis, although the significance of this in the context of JCA is uncertain. The clinical presentation of chlamydial arthritis can be very similar to JCA including the development of iridocyclitis. ${ }^{33}$ In a novel study from South Wales, a relationship was shown between influenza A infection in pregnancy and the subsequent development of polyarticular JCA in later childhood. ${ }^{34}$ This observation, yet to be confirmed by others, is a reminder that environmental exposure must also involve consideration of the intrauterine period. HLA B27 associated forms of JCA (or juvenile spondyloarthropathy) can be triggered by bacterial infections, as is the case with HLA B27 associated disease in adults. Consistent with this, an immunodominant epitope, GroEL of Escherichia coli HSPs, is one target of immune responsiveness in HLA B27 positive JCA. $^{35}$

\section{Summary}

In conclusion, it is evident that complex traits underlie the different forms of JCA, each one probably unique. Despite such differences, immune responses directed towards self antigens are likely to be central to the pathogenesis of each type of disease. This responsiveness will be determined by the particular array of genes present in an individual, that is, specific HLA alleles may favour the presentation of one peptide antigen over another to a specific $\mathrm{T}$ cell repertoire predetermined genetically at the thymic level (at least in part by HLA alleles also) and modified by the presence of null alleles in particular TCR families. A characteristic of such abnormal immune responses is the development of specific autoreactivity to self antigens such as HSP and nuclear DEK antigen, though whether the response to these antigens is disease promoting or protective is unknown in the setting of different forms of JCA. At least in the case of HSP, molecular mimicry with a homologous protein of an infecting organism has been postulated as a triggering event. It is conceivable that homology between other self and non-self proteins from different organisms may be found to be relevant and specific for the different forms of arthritis.

Given that autoreactive cells are likely to be deleted or suppressed in normal individuals, other genetic polymorphisms encoding mediators of the innate immune system have been implicated in disease susceptibility. The level of expression of particular alleles of cytokine genes (such as TNF- $\alpha$, IL-1, and IL-4) or of the apoptosis related Fas and Fas ligand molecules may create an inflammatory milieu which resets immune inflammatory responses at a level permitting autoreactive $T$ cell clones to persist, or become activated, with resulting autoimmunity.

The advent of more comprehensive approaches allowing genome-wide studies to be performed, the use of peptide libraries to look for relevant antigens ${ }^{36}$ and the further development of transgenic and gene knockout animal models is likely to herald greater understanding of the complex genetic traits and environmental triggers underlying the different forms of JCA. This, in turn, will enhance the prospect of developing more specific, effective and potentially curative treatments for these diseases. This work is supported in part by the Children's Hospital
Research Foundation of Cincinnati, the Schmidlapp FoundaResearch Foundation of Cincinnati, the Schmidlapp Founda-
tion and the National Institutes of Health (AR42632 and tion and the
AR44059). 
1 Fink CW. Proposal for the development of classification criteria for idiopathic arthritides of childhood. 7 Rheumatol 1995;22:156

2 Weyand CM, Goronzy, JJ. Pathogenesis of rheumatoid arthritis. Med Clin North Am 1997;81:29-55.

3 Rossen RD, Brewer EJ, Sharp RM, Ott J, Templeton JW. Familial rheumatoid arthritis; linkage of HLA to disease susceptibility locus in four families where proband presented with juvenile rheumatoid arthritis. $\mathcal{F}$ Clin Invest 1980;65:629-42. 4 Clemens LE, Albert E, Ansell BM. Sibling pairs affected by
chronic arthritis of childhood: evidence for a genetic predisposition. F Rheumatol 1985;12:108-13.

5 Moroldo M, Tague BL, Glass DN, Giannini EH. Juvenile rheumatoid arthritis in affected sib-pairs. Arthritis Rheum 1997 (in press).

6 Grom AA, Giannini EH, Glass DN. Juvenile rheumatoid arthritis and the trimolecular complex (HLA, T cell receptor, and antigen). Differences from rheumatoid arthritis. Arthritis Rheum 1994;37:601-7.

7 Nepom BS. The immunogenetics of juvenile rheumatoid arthritis. Rheum Dis Clin North Am 1991;17:825-42.

8 De Inocencio J, Giannini EH, Glass DN. Can genetic markDe Inocencio J, Giannini EH, Glass DN. Can genetic markers contribute to the classification of juvenile
arthritis. $\mathcal{F}$ Rheumatol 1993;20(suppl 40):12-8.

9 Schlosstein L, Terasaki PI, Bluestone R. High association of an HL-A antigen W27 with ankylosing spondylitis. $N$ Engl f Med 1973;288:704-6.

10 Murray KJ, Szer W, Grom AA, Giannini EH, Glass DN, Szer I. Antibodies to the $45 \mathrm{kDa}$ nuclear DEK antigen in pauciarticular onset JRA and iridocyclitis: selective association with an MHC gene. F Rheumatol 1997;24:560-7.

11 Haas JP, Nevinny-Stickel C, Schoenwald U, Truckenbrodt $\mathrm{H}$, Suschke J, Albert ED. Susceptible and protective major histocompatibility complex class II alleles in early-onset pauciarticular juvenile chronic arthritis. Hum Immunol 1994;41:225-33.

12 Haas JP, Kimura A, Truckenbrodt H, et al. Early-onset pauciarticular juvenile chronic arthritis is associated with a mutation in the Y box of the HLA-DQA 1 promoter. Tissue Antigens $1995 ; 45: 317-21$.

13 Davies EJ, Hutchings CJ, Hillarby MC, et al. HLA-DP does not contribute towards determining susceptibility to SLE Ann Rheum Dis 1994;53:188-190.

14 Albani S. Infection and molecular mimicry in autoimmune disease of childhood. Clin Exp Rheumatol 1994;12:535-41.

15 Giannini EH, Malagon CN, van Kerckhove C, et al. Longitudinal analysis of HLA associated risks for iridocyclitis in juvenile rheumatoid arthritis. I Rheumatol 1991;18:1394-7.

16 Pryhuber KG, Murray KJ, Donnelly P, et al. Polymorphism in the LMP2 gene influences disease susceptibility and severity in HLA-B27 associated juvenile rheumatoid arthritis. F Rheumatol 1996;23:747-52.

17 Donn RP, Davies, EJ, Holt PL, Thomson W, Ollier W. Increased frequency of TAP2B in early onset pauciarticular

18 Grom AA, Thompson SD, Luyrink L, Passo M, Choi E, Glass DN. Dominant $\mathrm{T}$ cell receptor $\mathrm{Vb} 14+$ clones in Glass DN. Dominant T cell receptor Vb 14+ clones in
juvenile rheumatoid arthritis. Proc Natl Acad Sci U S A juvenile rheumatoid

19 Walser-Kuntz DR, Weyand CM, Fulbright JW, Moore SB Goronzy JJ. HLA-DRB1 molecules and antigenic experience shape the repertoire of CD4 T cells. Hum Immunol 1995;44:203-9.
20 Luyrink L, Gabriel CA, Thompson SD, et al. Reduced expression of a human $\mathrm{Vb} 6.1$ T-cell receptor allele. Proc Natl Acad Sci U S A 1993;90:4369-73.

21 Shaw M, Clayton D, Atkinson SE, et al. Linkage of rheumatoid arthritis to the candidate gene NRAMP1 on chromosome 2q35. F Med Genet 1996;33:672-7.

22 Walley AJ, Cookson WO. Investigation of an interleukin-4 promoter polymorphism for associations with asthma and atopy. F Med Genet 1996;33:689-92.

23 Pociot F, D'Alfonso S, Compasso S, Scorza R, Richiardi $\mathrm{PM}$. Functional analysis of a new polymorphism in the human TNF alpha gene promoter. Scand 7 Rheumatol 1995;42:501-4.

24 McDowell TL, Symons JA, Ploski RP, Forre O, Duff GW. A genetic association between juvenile rheumatoid arthritis and a novel interleukin-1a polymorphism. Arthritis Rheum 1995;38:221-8.

25 Fearon DT, Locksley RM. The instructive role of innate immunity in the acquired immune response. Science 1996; 272:50-4.

26 Adachi M, Watange-Fukunaga R, Nagata S. Aberrant transcription caused by the insertion of an early transposable element in an intron of the Fas antigen gene of lpr mice. Proc Natl Acad Sci U S A 1993;90:1756-61.

27 Fisher G, Rosenberg F, Straus S. Dominant interfering Fas gene mutations impair apoptosis in a human autoimmune lymphoproliferative syndrome. Cell 1995;81:935-46.

28 Boivin GP, O'Toole BA, Ormsby IE, et al. Onset and progression of pathologic lesions in TGFb1-deficient mice. Am F Pathol 1995;146:276-88.

29 Davies JL, Kawaguchi Y, Bennet SI, et al. A genome wide search for human type 1 diabetes susceptibility genes. Nature 1994;371:130-6.

30 De Graeff-Meeder ER, Van Eden W, Rijkers GT, et al. Juvenile chronic arthritis: T cell reactivity to human HSP60 in patients with a favourable course of arthritis. $\mathcal{F}$ Clin Invest 1995;95:934-40.

31 Prakken ABJ, Van Eden W, Rijkers GT, et al. Autoreactivity to human heat-shock protein 60 predicts disease remission in oligoarticular juvenile rheumatoid arthritis. Arthritis Rheum 1996;39:1826-32.

32 Oen K, Fast M, Postl B. Epidemiology of juvenile rheumatoid arthritis in Manitoba, Canada, 1975-92: cycles in incidence. F Rheumatol 1995;22:745-50.

33 Maximov AA, Shaikow AV, Lovell DJ, Giannini EH, Soldatova SI. Chlamydial associated syndrome of arthritis and eye involvement in young children. $\mathcal{F}$ Rheumatol 1992;19. 1794-7.

34 Pritchard $\mathrm{MH}$, Matthews N, Munro J. Antibodies to influenza $\mathrm{A}$ in a cluster of children with juvenile chronic arthritis. Br f Rheumatol 1998;27:176-80.

35 Life P, Hassell A, Williams K, et al. Responses to Gram negative eneteric bacterial antigens by synovial $\mathrm{T}$ cells from patients with juvenile chronic arthritis: recognition of heat shock protein HSP60. I Rheumatol 1993;20:1388-96.

36 Sioud M, Forre O, Dybwad A. Selection of ligands for polyclonal antibodies from random peptide libraries: potential identification of (auto)antigens that may trigger $\mathrm{B}$ and $\mathrm{T}$ cell responses in autoimmune diseases. Clin Immunol Immunopathol 1996;79:105-14. 